\title{
Pyro-paraelectric and flexocaloric effects in barium strontium titanate: A first principles approach
}

Satyanarayan Patel, Aditya Chauhan, J. Cuozzo, S. Lisenkov, I. Ponomareva, and Rahul Vaish

Citation: Appl. Phys. Lett. 108, 162901 (2016);

View online: https://doi.org/10.1063/1.4947010

View Table of Contents: http://aip.scitation.org/toc/apl/108/16

Published by the American Institute of Physics

\section{Articles you may be interested in}

Multiple caloric effects in $\left(\mathrm{Ba}_{0.865} \mathrm{Ca}_{0.135} \mathrm{Zr}_{0.1089} \mathrm{Ti}_{0.8811} \mathrm{Fe}_{0.01}\right) \mathrm{O}_{3}$ ferroelectric ceramic

Applied Physics Letters 107, 042902 (2015); 10.1063/1.4927558

Ferroelectric, pyroelectric, and piezoelectric properties of a photovoltaic perovskite oxide

Applied Physics Letters 110, 063903 (2017); 10.1063/1.4974735

Combined intrinsic elastocaloric and electrocaloric properties of ferroelectrics

Journal of Applied Physics 121, 144102 (2017); 10.1063/1.4980098

How to measure the pyroelectric coefficient?

Applied Physics Reviews 4, 021303 (2017); 10.1063/1.4983118

Direct and indirect measurements on electrocaloric effect: Recent developments and perspectives

Applied Physics Reviews 3, 031102 (2016); 10.1063/1.4958327

Elastocaloric effect in ferroelectric ceramics

Applied Physics Letters 106, 172901 (2015); 10.1063/1.4919453

\section{Scilight}

Sharp, quick summaries illuminating the latest physics research

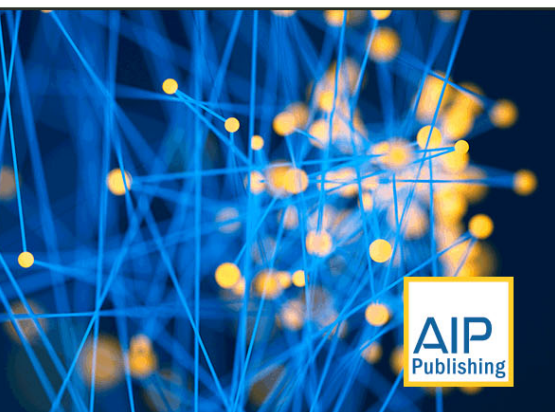




\title{
Pyro-paraelectric and flexocaloric effects in barium strontium titanate: A first principles approach
}

\author{
Satyanarayan Patel, ${ }^{1, a)}$ Aditya Chauhan, ${ }^{1,2, a)}$ J. Cuozzo, ${ }^{3}$ S. Lisenkov, ${ }^{3}$ I. Ponomareva, ${ }^{3}$ \\ and Rahul Vaish ${ }^{1, b)}$ \\ ${ }^{1}$ School of Engineering, Indian Institute of Technology Mandi, Mandi 175 001, Himachal Pradesh, India \\ ${ }^{2}$ Department of Materials Science and Metallurgy, University of Cambridge, CB3 OFS Cambridge, \\ United Kingdom \\ ${ }^{3}$ Department of Physics, University of South Florida, Tampa, Florida 33620, USA
}

(Received 28 November 2015; accepted 31 March 2016; published online 18 April 2016)

\begin{abstract}
Inhomogeneous strain allows the manifestation of an unexplored component of stress-driven caloric effect (flexocaloric effect) and enhanced pyroelectric performance, obtainable significantly beyond the Curie point. A peak temperature change of $1.5 \mathrm{~K}$ (at $289 \mathrm{~K}$ ) was predicted from first-principlesbased simulations for $\mathrm{Ba}_{0.5} \mathrm{Sr}_{0.5} \mathrm{TiO}_{3}$ under the application of a strain gradient of $1.5 \mu \mathrm{m}^{-1}$. Additionally, enhanced pyro-paraelectric coefficient (pyroelectric coefficient in paraelectric phase) and flexocaloric cooling $11 \times 10^{-4} \mathrm{C} \mathrm{m}^{-2} \mathrm{~K}^{-1}$ and $1.02 \mathrm{~K}$, respectively, could be obtained (at $330 \mathrm{~K}$ and $1.5 \mu \mathrm{m}^{-1}$ ). A comparative analysis with prevailing literature indicates huge untapped potential and warrants further research. Published by AIP Publishing. [http://dx.doi.org/10.1063/1.4947010]
\end{abstract}

According to a recent report by The Guardian, in about 25 years, the world would be spending more energy on creation of "artificial cold" than space heating or any other single application. ${ }^{1}$ Interestingly, while the concept is nearly 100 years old, the primary architecture has remained mostly unchanged and is based on vapour cycling of various halo-organic compounds. ${ }^{2}$ The process is crude and inefficient with a majority of the energy wasted as thermal pollution. ${ }^{2}$ Therefore, there is a need to replace the conventional system with a more efficient one. In this regards, solid state caloric effects are being rigorously investigated with hopes of realizing a refrigeration system capable of replacing the vapour-compression technology. ${ }^{3}$ Last decade has witnessed major progress in this field with magnetocaloric effect (MCE) and electrocaloric effect (ECE) as the two leading contenders. ${ }^{4}$ While recent developments in the field of ECE have displayed unforeseen "giant" cooling potential in solution-processed nano-composites, ${ }^{5} \mathrm{MCE}$ has established itself as a reliable form of refrigeration and specialized industrial deployment is already underway. ${ }^{2,6}$ However, the current state-of-art still suffers from several drawbacks which include relatively large magnetic field and rather narrow window of operating temperatures.

ECE uses polarization and depolarization of ferroelectric materials and offers higher operational temperatures and better tunability. ${ }^{3}$ Furthermore, recent reports indicate that these materials also have multi-caloric properties where several caloric effects can be combined to yield even better cooling efficiencies. ${ }^{7-9}$ Hence, devices based on ferroelectric materials may potentially overcome some of the shortcomings of the MCE-based devices. Ferroelectricity, pyroelectricity, and all associated effects are a direct consequence of the non-centrosymmetry of the crystal lattice. ${ }^{10}$ The pyroelectric effect decreases above the Curie point and disappears in centrosymmetric phase, which is unfavourable for

\footnotetext{
${ }^{\text {a) }}$ S. Patel and A. Chauhan contributed equally to this work.

${ }^{b)}$ Email: rahul@iitmandi.ac.in. Telephone: +91-1905-237921. Fax: +911905-237945.
}

the ECE. Interestingly, recent publications suggest that centrosymmetric dielectric materials are also able to display significant piezoelectric and pyroelectric effects. ${ }^{11-13}$ This entails linear coupling between inhomogeneous strain gradients and electric polarization. The phenomenon has been referred to as the flexoelectric effect. ${ }^{11}$ This flexoelectric effect originates from the inhomogeneous strain in a material owing to either its structure or the nature of stress applied. ${ }^{14}$ This leads to non-uniform deformation of the lattice, generating symmetry breaking. ${ }^{13-15}$ Consequently, such materials produce electrical charge when subjected to stress (flexoelectric effect) or mechanical straining when subjected to electric field (converse flexoelectric effect). ${ }^{13-15}$ The flexoelectric effect produces pyroelectricity in parelectric phase which is termed as pyro-paraelectric effect. ${ }^{12,16}$ Flexoelectric polarization in solid dielectrics can be written as ${ }^{11,13-15,17}$

$$
P_{i}=\mu_{i j k l} \frac{\partial \epsilon_{j k}}{\partial x_{l}} .
$$

Here, $P_{i}, \mu_{i j k l}, \epsilon_{j k}$, and $x_{l}$ represent flexoelectric polarization, flexoelectric coefficient, elastic strain, and Cartesian coordinate, respectively. Since $\mu_{i j k l}$ is a fourth-rank polar tensor, it can have non-zero components in all point group symmetries. ${ }^{15,17}$ This implies that even centro-symmetric structures can exhibit "effective" piezoelectric and pyroelectric effects. ${ }^{14,15,17}$ Literature review indicates that although flexoelectric effect can exist in essentially any solid dielectric material, the coupling coefficients are generally very small $\left(\sim 10^{-5}-10^{-4} \mu \mathrm{C} \mathrm{m}^{-1}\right){ }^{13,15,17}$ However, substantially larger coupling coefficients are found in ferroelectric materials. For example, in $\mathrm{Ba}_{\mathrm{x}} \mathrm{Sr}_{1-\mathrm{x}} \mathrm{TiO}_{3}$ (BST), the coupling coefficient is $\sim 100 \mu \mathrm{C} \mathrm{m}^{-1}$. ${ }^{17}$ This phenomenon is explained based on the dependence of flexoelectric coefficient on dielectric susceptibility of the material as follows: ${ }^{14,17}$

$$
\mu_{i j k l}=\chi_{i j} \gamma_{k l} \frac{e}{a}
$$


In Equation (2), $\chi_{i j}$ is the dielectric susceptibility and $\gamma_{k l}$ is a constant material tensor, while the symbols $e$ and $a$ represent the electron charge and atomic dimension of the unit cell. Thus, ferroelectric materials are expected to display much larger flexoelectric coefficients and stress induced polarization. Our group has previously demonstrated how change in stress-induced polarization in a bulk ferroelectric material can lead to inception of a "piezocaloric effect." ${ }^{8,18}$ Similarly, it is possible that polarization change and symmetry breaking due to inhomogeneous straining of the material could produce an analogous flexo-electro-thermal effect, or a flexocaloric effect (FCE). This caloric effect would contribute greatly to the multi-faceted caloric capacity of ferroelectric materials and would essentially help to expand the application of ferroelectric coolers to all possible regimes of operating temperature: potentially making ferroelectric materials suitable for a wider variety of cooling applications. This is, indeed, a lucrative prospect and demands serious attention. Hence, this letter aims to explore the FCE in BST ferroelectric material.

Flexoelectric effect in BST ceramics is well explored and documented in the literature. ${ }^{11,13-15,17}$ Many of these articles have focused on the effect of material morphology (size/shape) on flexoelectric response. ${ }^{12,13,17}$ The most investigated of the shapes are those of a bending beam and truncated pyramid. ${ }^{13-15,17}$ A graphical description of the same has been given in the inset of Figures 1(a) and 1(b), respectively. Let us now derive the expression for estimating the flexocaloric effect. Flexoelectric effect contributes a term into free energy expression that couples polarization and strain gradient as follows:

$$
G=G o+\gamma_{i j k l} P_{i} \frac{d \varepsilon_{j k}}{d x_{l}},
$$

where Go incorporates all the terms that are independent of the strain gradient, $\gamma_{i j k l}$ is the flexoelectric couplings, $P_{i}$ is the $i$ th Cartesian component of the polarization, and $\frac{d_{\varepsilon_{j k}}}{d x_{l}}$ is the gradient of $\varepsilon_{j k}$ component of the strain tensor along the $l$ th Cartesian direction. Equation (3) can be rewritten as

$$
G=G o+P_{i} E_{i}^{0},
$$

where

$$
E_{i}^{0}=\gamma_{i j k l} \frac{d \varepsilon_{j k}}{d x_{l}}
$$

is the thermal electric field. ${ }^{19}$ Maxwell relation for the flexocaloric temperature change can be derived similar to the ECE but replacing the applied electric field with the thermal electric field, $E_{0}$

$$
\Delta T_{\text {flexo }}=\int_{E_{i}^{0}(\text { init })}^{E_{i}^{0}(\text { fin })} \frac{T}{C}\left(\frac{\partial P_{i}}{\partial T}\right)_{E_{i}^{0}} d E_{i}^{0},
$$

where $C$ is the heat capacity. Note that the effect of strain gradient on ECE has been previously studied. ${ }^{20}$ However,
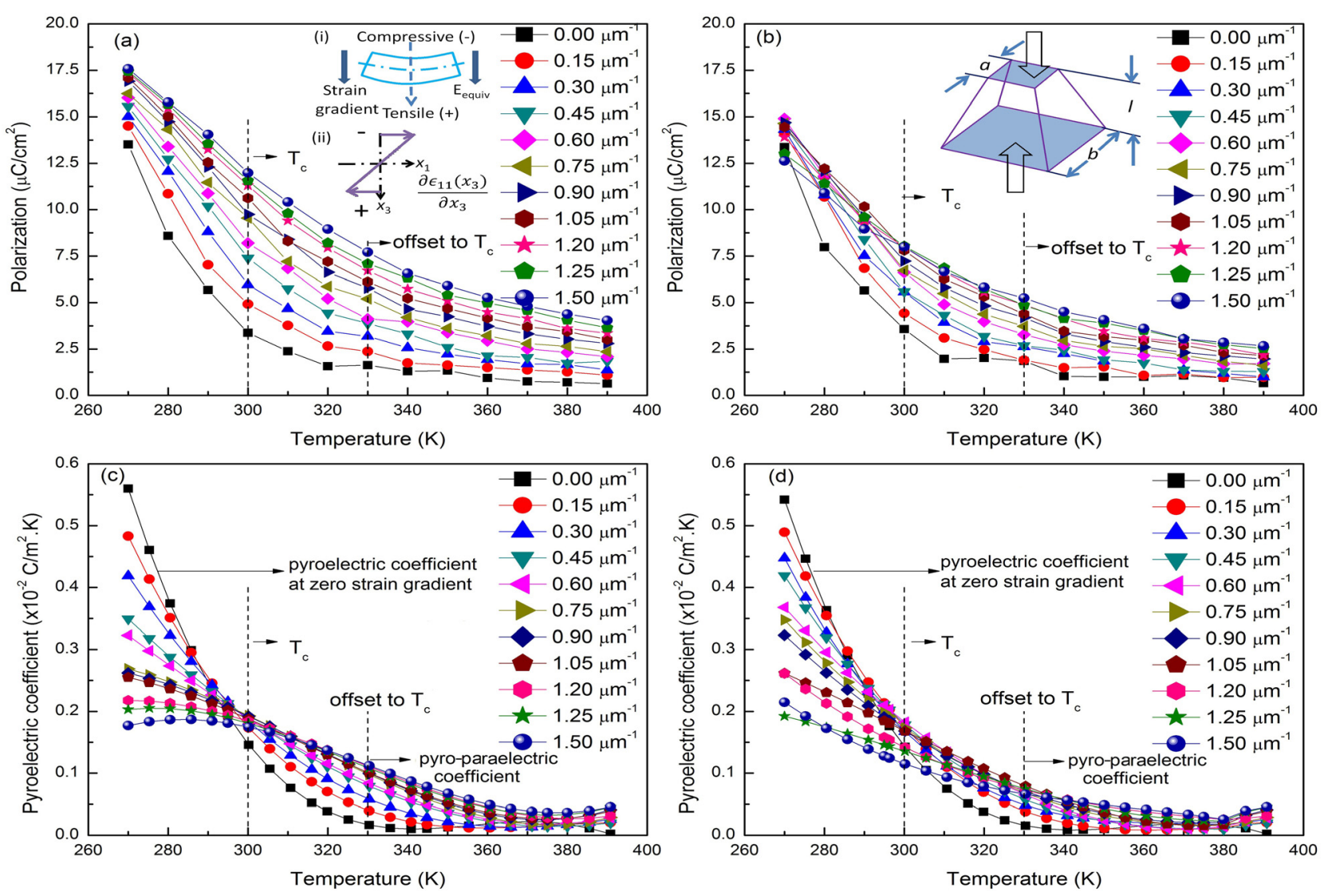

FIG. 1. (a) and (b) Polarization as a function of temperature under strain gradient of bent beam and truncated pyramid, respectively. Insets give the experimental setups that were simulated. (c) and (d) Pyroelectric coefficients calculated from the data given in (a) and (b), respectively. 
the flexocaloric effect proposed here is induced by strain gradient rather than applied electric field.

To estimate the flexocaloric effect in $\left(\mathrm{Ba}_{0.5} \mathrm{Sr}_{0.5}\right) \mathrm{TiO}_{3}$ ultrathin film, we employ the first-principles based approach as previously described. ${ }^{21}$ Here, we consider a film with 17 unit cells along the growth direction under short-circuit boundary conditions that are modeled by screening $98 \%$ of the polarization surface charge. The film is subjected to strain gradients $\partial \varepsilon_{z z} / \partial z$ or $\partial \varepsilon_{x x} / \partial z$ varying in magnitude from 0 to $1.5 \mu \mathrm{m}^{-1}$. Note that experimentally $\partial \varepsilon_{z z} / \partial z$ can be achieved using the truncated pyramid geometry, while $\partial \varepsilon_{x x} /$ $\partial z$ can be obtained using a bending setup. All the computational details are identical to the ones published in Ref. 21. Figures 1(a) and 1(b) show the temperature evolution of the out-of-plane polarization in the presence of $\partial \varepsilon_{z z} / \partial z$ or $\partial \varepsilon_{x x} / \partial z$ strain gradients of different magnitude. Figures 1(c) and 1(d) give the pyroelectric coefficients calculated from the data given in Figures 1(a) and 1(b), respectively. These dependences were obtained from the annealing simulations in which the film was slowly annealed from $390 \mathrm{~K}$ down to $270 \mathrm{~K}$ in steps of $5 \mathrm{~K}$.

To estimate the flexocaloric effect in the simulated films, we first compute the thermal electric field from Equation (5) using the first-principles flexoelectric couplings, $f_{i j j i}$, from Ref. 21. More precisely, $\gamma_{z z z z}=f_{z z z z} / 2=2.56 \mathrm{~V}$ and $\gamma_{z x x z}$ $=f_{z x x z} / 2=1.66 \mathrm{~V}$. This field is then used in Eq. (6) to estimate $\Delta T_{\text {flexo }}$ that is shown in Figures 2(a) and 2(b) for bending beam and truncated pyramid geometries, respectively. Furthermore, Figures 2(c) and 2(d) display the corresponding
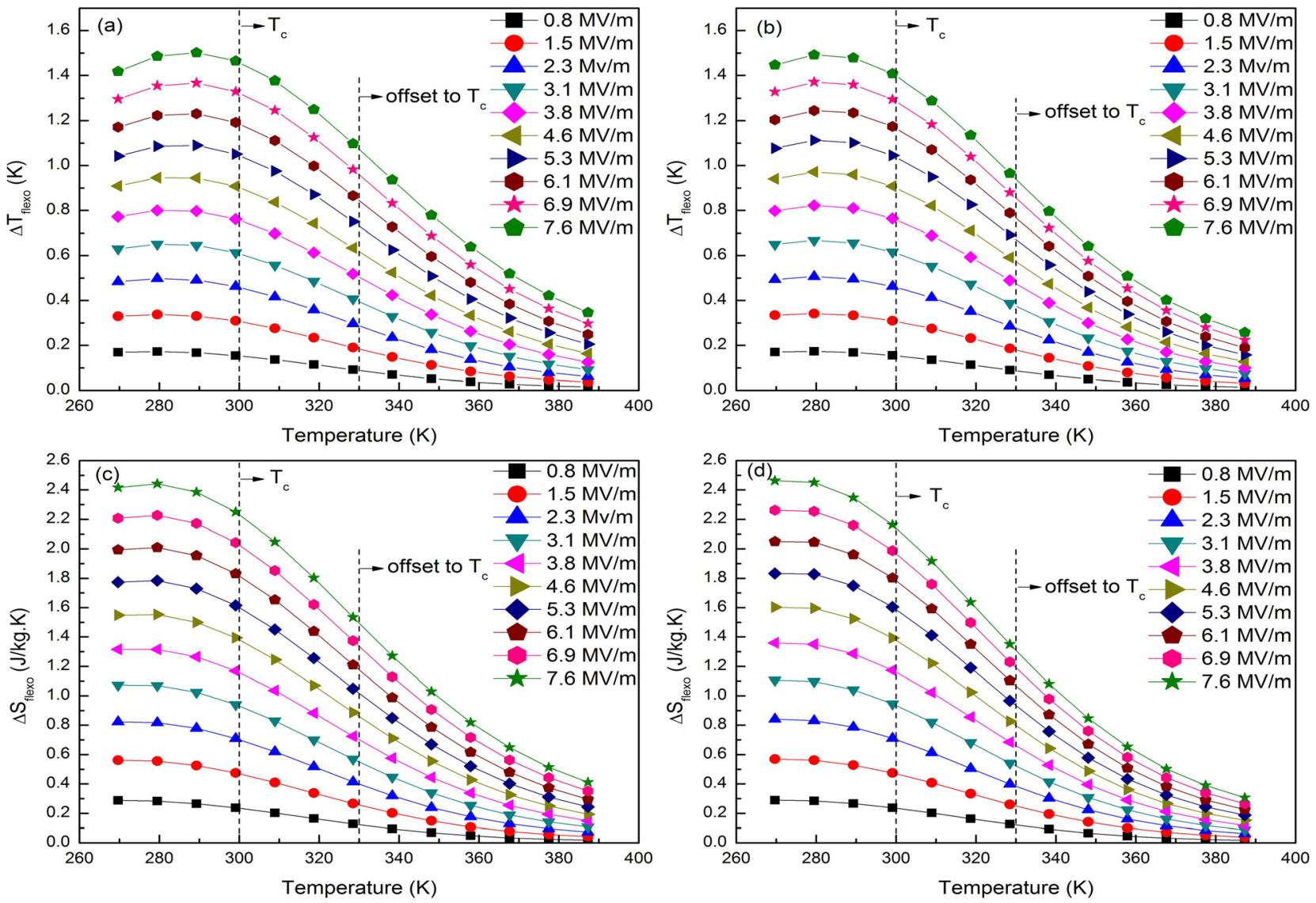

entropy change values for both the geometries. It is obtained using

$$
\Delta S_{\text {flexo }}=\int_{E_{i}^{0}(\text { init })}^{E_{i}^{0}(\text { fin })} \frac{1}{\rho}\left(\frac{\partial P_{i}}{\partial T}\right)_{E_{i}^{0}} d E_{i}^{0},
$$

where $\rho$ is density. The estimates suggest that the flexocaloric change in temperature can reach up to $1.5 \mathrm{~K}$ under the largest strain gradient of $1.5 \mu \mathrm{m}^{-1}$ considered in this work. Note that there is little difference in $\Delta T_{\text {fiexo }}$ under application of $\partial \varepsilon_{z z} / \partial z$ or $\partial \varepsilon_{x x} / \partial z$ strain gradients. The reason is that since the flexoelectric polarization is very small, the dominant contribution is from spontaneous polarization. It is also possible to estimate $\Delta T_{\text {fiexo }}$ using experimental data. To obtain experimental value for $E_{i}^{0}$, we notice that the flexoelectric polarization can be written as $^{13,21}$

$$
P_{m}^{f l e x o}=\chi_{m i} \varepsilon_{0} \gamma_{i j k l} \frac{\partial \varepsilon_{j k}}{\partial x_{l}}=\chi_{m i} \varepsilon_{0} E_{i}^{0}
$$

where $\chi_{m i} \varepsilon_{0} \gamma_{i j k l}=\mu_{m j k l}$ is the flexoelectric coefficient and $\chi_{m i}$ is the component of relative dielectric susceptibility tensor. It follows:

$$
E_{i}^{0}=\frac{P_{m}^{\text {flexo }}}{\chi_{m i} \varepsilon_{0}}=\frac{\mu_{m j k l}}{\chi_{m i}} \frac{1}{\varepsilon_{0}} \frac{\partial \varepsilon_{j k}}{\partial x_{l}}
$$

FIG. 2. (a) and (b) Flexocaloric adiabatic temperature change as a function of operating temperature for bending beam and truncated pyramid geometry, respectively. (c) and (b) Corresponding volume specific entropy change. 
For $\left(\mathrm{Ba}_{0.67} \mathrm{Sr}_{0.33}\right) \mathrm{TiO}_{3}$ ceramics, $\mu_{\mathrm{zxxz}} / \chi \sim 4 \mathrm{nC} \mathrm{m}^{-2} \cdot{ }^{13}$ For the largest strain gradient of $0.001 \mathrm{~m}^{-1}$ (applied to the bulk ceramics in Ref. 13), we obtained $\mathrm{E}^{0} \sim 0.5 \mathrm{~V} \mathrm{~m}^{-1}$ that is too small to observe a flexocaloric effect. However, for the strain gradients of up to $0.1 \mu \mathrm{m}^{-1}$, the thermal electric field can reach $450 \mathrm{kV} / \mathrm{cm}^{-1}$ which is in the range of the fields associated with giant electrocaloric effects.

A comparison of the present results with those reported in the literature is given in Tables I and II. Table I lists the pyroelectric coefficients of some known materials, while Table II lists various caloric effects reported in the literature for BST. Examination of Table I reveals that pyroparaelectric coefficient (pyroelectric coefficient above the offset of $\mathrm{T}_{\mathrm{c}}$ ) reported in this study is of the same order as other pyroelectric materials. This in itself is a surprising result as it suggests that a centro-symmetric structure

TABLE I. Pyroelectric coefficient of selected materials near room temperature.

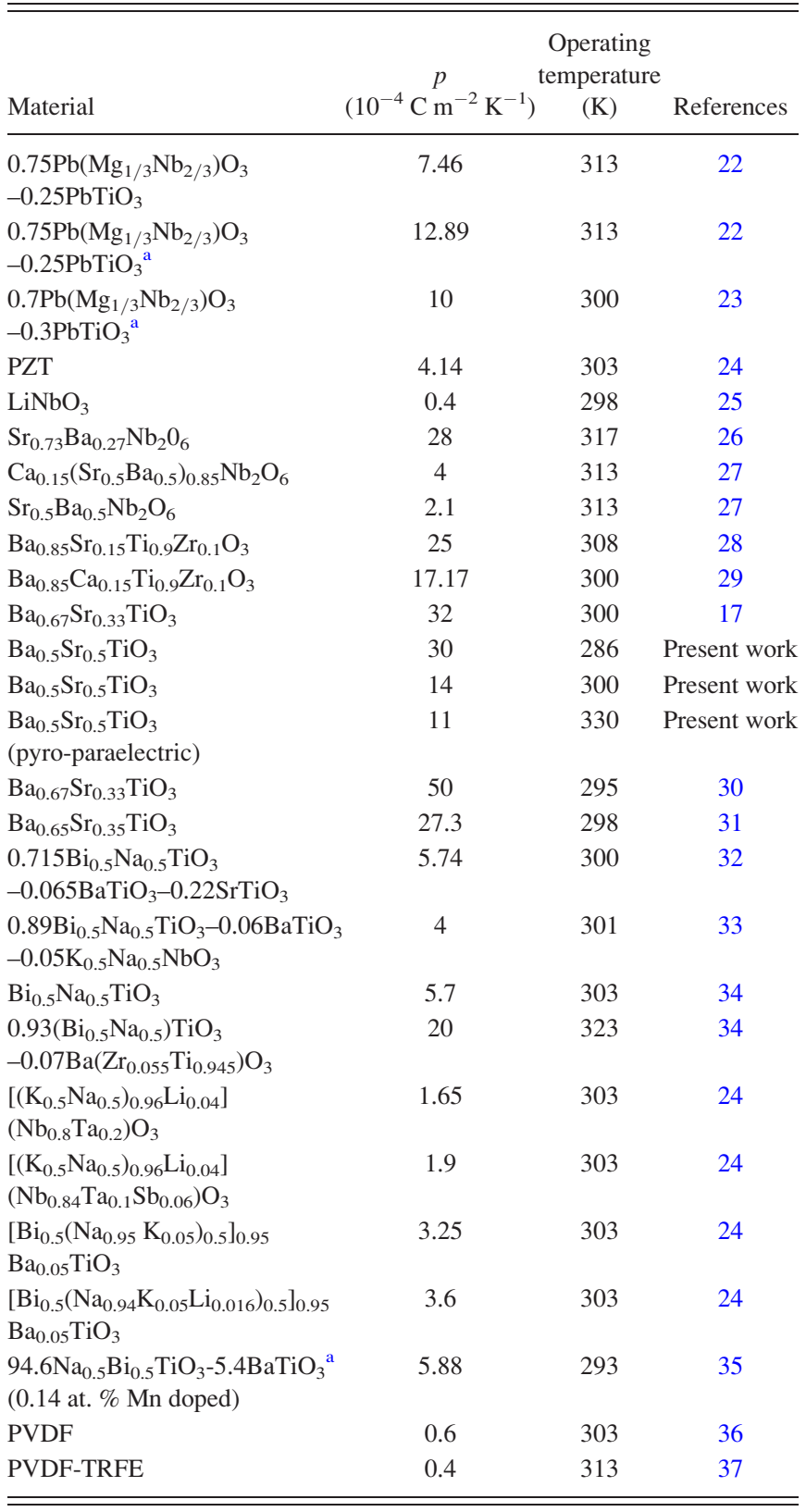

${ }^{\mathrm{a}}$ Single crystal. possesses pyroelectric performance similar to most commercially important materials. Furthermore, whereas the conventional pyroelectric materials lose their functionality above the Curie temperature, the values reported in this study could be easily sustained well beyond the Curie point. Here, it is important to note that the pyroelectric coefficient is almost zero at or above $330 \mathrm{~K}$ under zero strain gradients. However, due to flexoelectric effect, a pyro-paraelectric coefficient of $11 \times 10^{-4} \mathrm{C} / \mathrm{m}^{-2} \mathrm{~K}^{-1}$ (at $330 \mathrm{~K}$ ) could be obtained under strain gradient of $1.5 \mu \mathrm{m}^{-1}$. This pyroelectric effect (pyro-paraelectric effect) is purely due to flexoelectric polarization contribution. Such a finding may allow the fabrication of pyroelectric detectors/transducers with improved efficiency and thermally invariant performance over a wider range of temperature. Furthermore, such performance would be readily achievable by simply prestressing the material in a proper manner.

Similarly, data presented in Table II allows easy discerning of the fact that the flexocaloric performance of BST is at par with most other caloric effects reported in the literature for the same family of materials. The peak flexocaloric effect of $1.5 \mathrm{~K}$ is obtainable at a temperature of $289 \mathrm{~K}$, while a significant $\sim 1 \mathrm{~K}$ effect can still be observed at a much elevated temperature of $330 \mathrm{~K}$ at strain gradient of $1.5 \mu \mathrm{m}^{-1}$. This FCE at $330 \mathrm{~K}$ is again pure contribution of flexoelectric polarization only. The adiabatic temperature change capacity increases with increasing strain gradient and reaches a maximum at $1.5 \mu \mathrm{m}^{-1}$ corresponding to a thermal electric field value of $7.6 \mathrm{MV} \mathrm{m}^{-1}$. The same can be observed from plots presented in Figures 2(a) and 2(b) and the corresponding entropy change in Figures 2(c) and 2(d). At this point, it becomes imperative to mention that the reported values are for a limited strain gradient distribution and for a given composition $\left(\mathrm{Ba}_{0.5} \mathrm{Sr}_{0.5}\right) \mathrm{TiO}_{3}$ which is centrosymmetric at room temperature. It is highly possible that larger values could be established using an optimized combination of strain gradient and material chemistry. The reported $\Delta T_{\text {flexo }}$ is the same for both the employed geometries of bent beam and truncated pyramid. However, from an engineering perspective, a bending beam would be easier to incorporate and actuate in a realistic device setup. Additionally, as made evident from the results, this configuration could also be employed to fabricate superior performing pyroelectric detectors. It is the authors' opinion that there is huge untapped potential within this field and hence further research is warranted. Such efforts must be duly supported by experimental evidence, and these will be undertaken in subsequent studies.

In conclusion, the authors predicted a pyroelectric (pyro-paraelectric) and flexocaloric effect in centrosymmetric BST structures. The results suggest that certain ferroelectric materials could be used to produce cooling well above their Curie temperature by suitably employing the flexoelectric effect. Furthermore, it was observed that these materials under optimized conditions of pre-stress could outperform some commercial pyroelectric materials, including lead-based compositions. Temperature dependence of the flexocaloric effect has been explored which indicates peak performance at $289 \mathrm{~K}$. This study reports promising cooling effect (above the Curie temperature) in ferroelectric materials. 
TABLE II. A comparative analysis of the various caloric effects reported in literature for $\mathrm{Ba}_{x} \mathrm{Sr}_{1-\mathrm{x}} \mathrm{TiO}_{3}$ family of ferroelectric ceramics. IM-Indirect measurement, DM-Direct measurements, FP-First principal, and SS-Simulation study.

\begin{tabular}{|c|c|c|c|c|c|c|}
\hline Material & Type & $\Delta \mathrm{T}(\mathrm{K})$ & $\mathrm{T}_{\text {operating }}(\mathrm{K})$ & Input Parameter & Method & References \\
\hline $\mathrm{Ba}_{0.65} \mathrm{Sr}_{0.35} \mathrm{TiO}_{3}(\mathrm{SPS})$ & Bulk & 2.1 & 303 & $9 \mathrm{MV} \mathrm{m}^{-1}$ & IM & 38 \\
\hline $\mathrm{Ba}_{0.7} \mathrm{Sr}_{0.3} \mathrm{TiO}_{3}^{\mathrm{a}}$ & Thin film & 1.56 & 278 & $35 \mathrm{MV} \mathrm{m}^{-1}$ & $\mathrm{IM}$ & 39 \\
\hline $\mathrm{Ba}_{0.8} \mathrm{Sr}_{0.2} \mathrm{TiO}_{3}{ }^{\mathrm{b}}$ & Thin film & 1.75 & 314 & $35 \mathrm{MV} \mathrm{m}^{-1}$ & IM & 39 \\
\hline $\mathrm{Ba}_{0.9} \mathrm{Sr}_{0.1} \mathrm{TiO}_{3}{ }^{\mathrm{c}}$ & Thin film & 1.9 & 350 & $35 \mathrm{MV} \mathrm{m}^{-1}$ & IM & 39 \\
\hline Compositionally gradient film of $\mathrm{a}, \mathrm{b}$ and $\mathrm{c}$ & Thin film & 3.2 & 290 & $35 \mathrm{MV} \mathrm{m}^{-1}$ & $\mathrm{IM}$ & 39 \\
\hline $\mathrm{Ba}_{0.7} \mathrm{Sr}_{0.3} \mathrm{TiO}_{3}(\mathrm{SPS})$ & Bulk & 1.8 & 310 & $9 \mathrm{MV} \mathrm{m}^{-1}$ & $\mathrm{IM}$ & 40 \\
\hline$(\mathrm{Ba}, \mathrm{Sr}) \mathrm{TiO}_{3}$ & $\ldots$ & 20 & $\ldots$ & & SS & 41 \\
\hline $\mathrm{Ba}_{0.73} \mathrm{Sr}_{0.27} \mathrm{TiO}_{3}$ & Bulk & 1 & 298 & $1.5 \mathrm{MV} \mathrm{m}^{-1}$ & DM & 42 \\
\hline $\mathrm{Ba}_{0.67} \mathrm{Sr}_{0.33} \mathrm{TiO}_{3}$ & Bulk & 0.4 & 298 & $1 \mathrm{MV} \mathrm{m}^{-1}$ & $\mathrm{DM}$ & 43 \\
\hline $\mathrm{Ba}_{0.5} \mathrm{Sr}_{0.5} \mathrm{TiO}_{3}$ & $\ldots$ & 16 & 300 & $50 \mathrm{MV} \mathrm{m}^{-1}$ & FP & 44 \\
\hline $\mathrm{Ba}_{0.5} \mathrm{Sr}_{0.5} \mathrm{TiO}_{3}$ (Elastocaloric) & $\ldots$ & 8 & 300 & $1 \mathrm{GPa}$ & FP & 9 \\
\hline $\mathrm{Ba}_{0.5} \mathrm{Sr}_{0.5} \mathrm{TiO}_{3}$ (Flexocaloric) & $\ldots$ & 1.5 & 289 & $1.5 \mu \mathrm{m}^{-1}$ & FP & Present work \\
\hline $\mathrm{Ba}_{0.5} \mathrm{Sr}_{0.5} \mathrm{TiO}_{3}$ (Flexocaloric) & $\ldots$ & 1.02 & 330 & $1.5 \mu \mathrm{m}^{-1}$ & FP & Present work \\
\hline
\end{tabular}

One of the authors (R.V.) acknowledges support from the Indian National Science Academy (INSA), Delhi, India, through a grant by the Department of Science and Technology (DST), Delhi, under INSPIRE faculty award2011 (ENG-01) and INSA Young Scientists Medal-2013. A.C. would like to acknowledge the support of SERB, India in the form of Cambridge India Ramanujan fellowship. I.P. acknowledges support from the National Science Foundation Grant Nos. DMR-1250492 and MRI CHE-1531590.

${ }^{1}$ J. Henley, "World set to use more energy for cooling than heating," The Guardian (26 October 2015), available at http://www.theguardian. com/environment/2015/oct/26/cold-economy-cop21-global-warming-carbonemissions (last accessed 26 November 2015).

${ }^{2}$ A. Kitanovski, U. Plaznik, U. Tomc, and A. Poredoš, Int. J. Refrig. 57, 288 (2015).

${ }^{3}$ M. Ožbolt, A. Kitanovski, J. Tušek, and A. Poredoš, Int. J. Refrig. 40, 174 (2014).

${ }^{4}$ M. Valant, Prog. Mater. Sci. 57(6), 980 (2012).

${ }^{5}$ G. Zhang, X. Zhang, T. Yang, Q. Li, L.-Q. Chen, S. Jiang, and Q. Wang, ACS Nano 9(7), 7164 (2015).

${ }^{6}$ D. Silva, B. Bordalo, A. Pereira, J. Ventura, and J. Araújo, Appl. Energy 93, 570 (2012).

${ }^{7}$ A. Chauhan, S. Patel, and R. Vaish, Acta Mater. 89, 384 (2015); A. Chauhan, S. Patel, and R. Vaish, Appl. Phys. Lett. 106(17), 172901 (2015).

${ }^{8}$ S. Patel, A. Chauhan, and R. Vaish, Appl. Phys. Lett. 107(4), 042902 (2015).

${ }^{9}$ S. Lisenkov and I. Ponomareva, Phys. Rev. B 86(10), 104103 (2012).

${ }^{10}$ R. Baggio, M. De Benyacar, B. Perazzo, and P. De Perazzo, Acta Crystallogr., Sect. B: Struct. Sci. 33(11), 3495 (1977).

${ }^{11}$ A. Biancoli, C. M. Fancher, J. L. Jones, and D. Damjanovic, Nat. Mater. 14(2), 224 (2015).

${ }^{12}$ H.-A. Chin, S. Mao, B. L. Visweswaran, K. K. Ohemeng, S. Wagner, P. K. Purohit, and M. C. McAlpine, paper presented at the SPIE Smart Structures and Materials+ Nondestructive Evaluation and Health Monitoring (2015).

${ }^{13}$ L. E. Cross, J. Mater. Sci. 41(1), 53 (2006).

${ }^{14}$ X. Jiang, W. Huang, and S. Zhang, Nano Energy 2(6), 1079 (2013).

${ }^{15}$ W. Ma, Phys. Status Solidi B 247(1), 213 (2010).

${ }^{16}$ H.-A. Chin, S. Mao, C.-T. Huang, K. K. Ohemeng, S. Wagner, P. K. Purohit, and M. C. McAlpine, Extreme Mech. Lett. 2, 20 (2015).

${ }^{17}$ G. Bai, Z. Liu, Q. Xie, Y. Guo, W. Li, and X. Yan, AIP Adv. 5(9), 097117 (2015).

${ }^{18}$ A. Chauhan, S. Patel, and R. Vaish, Acta Mater. 97, 17 (2015).
${ }^{19}$ M. E. Lines and A. M. Glass, Principles and applications of ferroelectrics and related materials (Oxford University Press, 1977).

${ }^{20}$ A. Starkov and I. Starkov, J. Exp. Theor. Phys. 119(2), 258 (2014).

${ }^{21}$ I. Ponomareva, A. Tagantsev, and L. Bellaiche, Phys. Rev. B 85(10), 104101 (2012).

${ }^{22}$ G. Sebald, L. Seveyrat, D. Guyomar, L. Lebrun, B. Guiffard, and S. Pruvost, J. Appl. Phys. 100(12), 124112 (2006).

${ }^{23}$ Y. J. Ko, Y. K. Park, B. K. Yun, M. Lee, and J. H. Jung, Curr. Appl. Phys. 14(11), 1486 (2014).

${ }^{24}$ S. T. Lau, C. H. Cheng, S. H. Choy, D. M. Lin, K. W. Kwok, and H. L. W. Chan, J. Appl. Phys. 103(10), 104105 (2008).

${ }^{25}$ A. Savage, J. Appl. Phys. 37(8), 3071 (1966).

${ }^{26}$ A. M. Glass, J. Appl. Phys. 40(12), 4699 (1969).

${ }^{27}$ J. Zhang, X. Dong, F. Cao, S. Guo, and G. Wang, Appl. Phys. Lett. 102(10), 102908 (2013).

${ }^{28}$ S. Patel, A. Chauhan, and R. Vaish, Solid State Sci. 52, 10 (2016).

${ }^{29}$ Y. Shanshan, R. Wei, J. Hongfen, W. Xiaoqing, S. Peng, X. Dezhen, R. Xiaobing, and Y. Zuo-Guang, J. Phys. D: Appl. Phys. 45(19), 195301 (2012).

${ }^{30}$ G. Zhang, S. Jiang, Y. Zeng, Y. Zhang, Q. Zhang, and Y. Yu, J. Am. Ceram. Soc. 92(12), 3132 (2009).

${ }^{31}$ C. G. Wu, Y. R. Li, J. Zhu, X. Z. Liu, and W. L. Zhang, J. Appl. Phys. 105(4), 044107 (2009).

${ }^{32}$ S. Patel, A. Chauhan, S. Kundu, N. A. Madhar, B. Ilahi, R. Vaish, and K. B. R. Varma, AIP Adv. 5(8), 087145 (2015).

${ }^{33}$ F. Gao, X. Dong, C. Mao, H. Zhang, F. Cao, and G. Wang, J. Appl. Phys. 110(9), 094109 (2011).

${ }^{34}$ F. Guo, B. Yang, S. Zhang, F. Wu, D. Liu, P. Hu, Y. Sun, D. Wang, and W. Cao, Appl. Phys. Lett. 103(18), 182906 (2013).

${ }^{35}$ R. Sun, J. Wang, F. Wang, T. Feng, Y. Li, Z. Chi, X. Zhao, and H. Luo, J. Appl. Phys. 115(7), 074101 (2014).

${ }^{36}$ T. Kaura, R. Nath, and M. M. Perlman, J. Phys. D: Appl. Phys. 24(10), 1848 (1991).

${ }^{37}$ B. Ploss, F. Shin, H. Chan, and C. Choy, IEEE Trans. Dielectr. Electr. Insul. 7(4), 517 (2000).

${ }^{38}$ X. Q. Liu, T. T. Chen, Y. J. Wu, and X. M. Chen, J. Am. Ceram. Soc. 96(4), 1021 (2013).

${ }^{39}$ B. Liu, J. Wang, X. Zhong, K. Huang, B. Li, F. Wang, J. Xie, and Y. Zhou, RSC Adv. 4(47), 24533 (2014).

${ }^{40}$ X. Q. Liu, T. T. Chen, M. S. Fu, Y. J. Wu, and X. M. Chen, Ceram. Int. 40(7, Part B), 11269 (2014).

${ }^{41}$ S. F. Karmanenko, O. V. Pakhomov, A. M. Prudan, A. S. Starkov, and A. Eskov, J. Eur. Ceram. Soc. 27(8-9), 3109 (2007).

${ }^{42}$ G. Lin, X. Xiong, J. Zhang, and Q. Wei, J. Therm. Anal. Calorim. 81(1), 41 (2005).

${ }^{43}$ M. G. Hilt, "A solid-state heat pump using electrocaloric ceramic elements," Ph.D. thesis, The Pennsylvania State University, 2009.

${ }^{44}$ S. Lisenkov and I. Ponomareva, Phys. Rev. B 80(14), 140102 (2009). 